\title{
Design, construction and evaluation of multi- epitope antigens for diagnosis of Lyme disease
}

\author{
Eva Schreterova', Mangesh Bhide ${ }^{1,2}$, Lenka Potocnakova', Lucia Borszekova Pulzova' \\ ${ }^{1}$ Laboratory of Biomedical Microbiology and Immunology, Department of Microbiology and Immunology, University of \\ Veterinary Medicine and Pharmacy, 04181 Kosice, Slovakia \\ ${ }^{2}$ Institute of Neuroimmunology, Slovak Academy of Sciences, 84510 Bratislava, Slovakia
}

Schreterova E, Bhide M, Potocnakova L, Borszekova Pulzova L. Design, construction and evaluation of multi-epitope antigens for diagnosis of Lyme disease. Ann Agric Environ Med. 2017; 24(4): 696-701. doi: 10.26444/aaem/80699

\begin{abstract}
Introduction and objective. Lyme disease (LD) is the most common vector-borne disease in the temperate zone of the Northern Hemisphere. Diagnosis of LD is mainly based on clinical symptoms supported with serology (detection of antiBorrelia antibodies) and is often misdiagnosed in areas of endemicity.

Materials and method. In this study, the chimeric proteins (A/C-2, A/C-4 and A/C-7.1) consisting of B-cell epitopes of outer surface proteins OspA and OspC from Borrelia genospecies prevalent in Eastern Slovakia, were designed, over-expressed in E. coli, and used to detect specific anti-Borrelia antibodies in serologically characterized sera from patients with Lyme-like symptoms to evaluate their diagnostic potential.

Results. Results showed that chimeras vary in their immuno-reactivity when tested with human sera. Compared with the results obtained from a two-tier test, the application of recombinant multi-epitope chimeric proteins as diagnosis antigens, produced fair agreement in the case of $A / C-2(0.20<\kappa<0.40)$ and good agreement $(0.60<\kappa<0.80)$ when $A / C-7.1$ was used as capture antigen. Chimera A/C-4 were excluded from further study due to loss of reactivity with OspA-specific antibodies. Conclusions. The combination of specific B-cell epitopes from OspA and OspC proteins may improve the diagnostic accuracy of serologic assays, but further studies are required to address this hypothesis.
\end{abstract}

Key words

Lyme disease, epitope, chimera, ELISA, Slovakia

\section{INTRODUCTION}

Lyme disease $(\mathrm{LD})$ is a multisystem and multistage infection caused by three major Borrelia genospecies. Diagnosis of LD is based on clinical symptoms and the two-tier detection of anti-Borrelia antibodies. First, an ELISA is performed, followed by a Western blot to confirm the results from the first serology, as well as to identify the infecting Borrelia species [1]. Unfortunately, the assays available commercially and assays developed in many laboratories worldwide have wide divergent sensitivity and specificity. Whole-cell antigens, purified antigens (flagellar components), whole-cell antigens combined with recombinant antigens and exclusively recombinant antigens (e.g. C6 peptide, P39) are being used in serodiagnosis of LD [2]. The vast majority of borrelial proteins are cross-reactive [3]. The complexity of the antigenic composition among the Borrelia genospecies and differential expression of proteins in host and vector (temporal and spatial antigenic variability) has posed challenges for the serodiagnosis of LD. Therefore, the optimal combination of the most specific epitopes could increase the discrimination abilities of serodiagnostic tests.

Among numerous antigens of Borrelia, the outer surface proteins OspA to OspG and the flagellin are important in diagnostics [4]. OspA protein has been detected in the serum from patients diagnosed with early LD [5]. Elevated levels of anti-OspA antibodies have also been shown in the sera

Address for correspondence: Lucia Borszekova Pulzova, Laboratory of Biomedical Microbiology and Immunology, Department of Microbiology and Immunology, University of Veterinary Medicine and Pharmacy, Komenskeho 73, 04181, Kosice, Slovakia

E-mail:pulzova@gmail.com

Received: 20.06.2017; accepted: 21.11.2017; first published: 18.12.2017 of patients with severe arthritis and neurological symptoms (late LD) [6]. An important protective B-cell epitope of OspA, LA-2 epitope, was identified earlier by murine monoclonal antibody [7]. Besides OspA, outer surface protein OspC is a promising candidate for the construction of an $\mathrm{LD}$ diagnostic marker due to its high immunologic potential and its early expression during LD infection [8]. Six OspC B-cell epitopes were identified recently using phage display [9].

Although the genospecies of $B$. burgdorferi sensu lato complex express a generally similar repertoire of antigens, significant sequence variation both between and within species do occur [10]. This variation complicates the development of single immunodiagnostic assay for all major Borrelia genospecies [4]. The development of assays based on antigens specific for the genospecies observed in a defined geographic area represents a promising approach for improvement in the accuracy and reliability of immunoassays [11]. In the presented study, chimeric proteins were produced, consisting of OspA and OspC B-cell epitopes for Eastern Slovakia specific genospecies and their diagnostic potential was evaluated and compared to the commercial two-tier test. Application of A/C-2 produced fair agreement $(0.20<\kappa<0.40)$ and good agreement $(0.60<\kappa<0.80)$ when A/C7.1 was used as the capture antigen.

\section{MATERIALS AND METHOD}

Design and cloning of recombinant chimeras. The OspC epitopes of B. burgdorferi SKT-2 (AY597037.1) were mapped with the help of phage display in our previous study [9]. Epitopes in other genospecies (B. bavariensis SKT-7.1 GU906888.1 and B. afzelii strain SKT-4 AY518771.1; both 


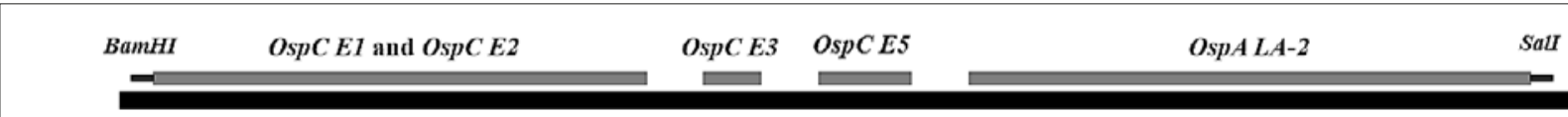

Synthetic gene

Figure 1. Strategy used to design synthetic genes

strains isolated in Slovakia) were defined with multiple alignment of OspC sequences using SKT-2 as reference. The LA-2 epitope of OspA was identified by murine monoclonal antibody LA-2 [7]. This epitope was defined in OspA of borrelial strains specific for Slovakia (SKT-2 strain AY597021.1, B. bavariensis strain SKT-7.1 AY597026.1 and B. afzelii strain SKT-4 AY597023.1) with multiple alignment, using OspA from B31 as a reference sequence.

Synthetic genes $(a / c-2-381 \mathrm{bp}, a / c-7.1-414 \mathrm{bp}$ and $a / c-4$ - $384 \mathrm{bp}$ ) were constructed consisting of BamHI restriction site on $5^{\prime}$ end, followed by four OspC epitopes (OspC E1, OspC E2, OspC E3 and OspC E5) and OspA epitope LA2. Between each epitope the flexible (GSGSG) linker was inserted. The epitopes OspC E1 and OspC E2 were present in tandem. At the 3' end of the synthetic gene the stop codon was followed by SalI restriction site. Synthetic gene sequence was assembled in silico with the help of Geneious pro software (Biomatters, New Zealand) and custom synthesized (Life Technologies). The strategy used for designing of a chimeric gene is shown in Figure 1. After digestion with restriction enzymes, chimeric genes were gel purified and ligated into vector pQE-mCHERRY-GFP (in house modified vector). Recombinant vectors were transformed in $E$. coli (XL-1 blue) and transformants were selected by plating on kanamycin $(25 \mu \mathrm{g} / \mathrm{ml})$ and carbenicillin $(50 \mu \mathrm{g} / \mathrm{ml})$ containing LB plates (1\% tryptone, $0.5 \%$ yeast extract, $1 \% \mathrm{NaCl}$ and $1 \%$ agar). DNA from carbenicillin-resistant colonies was isolated, amplified with vector specific primers (sense CGCATCACCATCACCATCACG and antisense ACCAAAATTGGGACAACACCAGTG), and amplicons were characterized by sequencing.

Protein production and purification. A single colony for each chimera was grown in $50 \mathrm{ml}$ of TB medium (1.5\% peptone, $3 \%$ yeast extract, $1.25 \% \mathrm{NaCl}, 0.25 \% \mathrm{MgCl}_{2} \cdot 6 \mathrm{H}_{2} \mathrm{O}$ and $0.735 \%$ glycerol) supplemented with metal mix $\left(0.17 \mathrm{M} \mathrm{KH}_{2} \mathrm{PO}_{4}\right.$ and $\left.0.72 \mathrm{M} \mathrm{K}_{2} \mathrm{HPO}\right)$, carbenicillin $(50 \mu \mathrm{g} / \mathrm{ml})$, kanamycin $(25 \mu \mathrm{g} /$ $\mathrm{ml}$ ) and $1 \%$ glucose at $37^{\circ} \mathrm{C}$ with constant shaking $300 \mathrm{rpm}$ to the $\mathrm{OD}_{600} 6.0$. Cells were harvested by centrifugation $(6,000$ $\mathrm{rpm}, 10 \mathrm{~min}, 20^{\circ} \mathrm{C}$ ) and resuspended in $50 \mathrm{ml}$ of TB medium without glucose. Recombinant protein expression was induced by adding IPTG (isopropyl- $\beta$-d-thiogalactopyranoside) to a final concentration of $1.0 \mathrm{mM}$ and cells were grown for an additional $12 \mathrm{hrs}$ at $18^{\circ} \mathrm{C}$ with constant shaking. Cells were harvested by centrifugation and resuspended in denaturative lysis buffer $\left(50 \mathrm{mM} \mathrm{NaH}_{2} \mathrm{PO}_{4} .2 \mathrm{H}_{2} \mathrm{O}, 300 \mathrm{mM} \mathrm{NaCl}, 8 \mathrm{M}\right.$ urea, pH 7.5) supplemented with DNase I (Fermentas) and ProteoBlock protease inhibitor cocktail (Fermentas). Repeated freeze-thaw cycles ( 5 cycles) and sonication on ice (5 pulses of 10 seconds, amplitude of 50 at frequency of $20 \mathrm{kHZ}$ ) were used to lyse cells. Supernatant containing chimeric proteins was cleared by repeated centrifugation at $14,000 \mathrm{rpm}$ for 20 $\mathrm{min}$. The chimeric proteins were purified under denaturative conditions using Ni-NTA agarose beads (Qiagen, USA) and eluted with $300 \mathrm{mM}$ immidazole in elution buffer $(50 \mathrm{mM}$ $\mathrm{NaH}_{2} \mathrm{PO}_{4} \cdot 2 \mathrm{H}_{2} \mathrm{O}, 300 \mathrm{mM} \mathrm{NaCl}, 8 \mathrm{M}$ urea, $\left.\mathrm{pH} 7.5\right)$. The eluate was dialyzed against PBS (137mM NaCl, 2.7mM KCl, $4.3 \mathrm{mM}$ $\left.\mathrm{Na}_{2} \mathrm{HPO}_{4} .12 \mathrm{H}_{2} \mathrm{O}, 1.47 \mathrm{mM} \mathrm{KH} \mathrm{PO}_{4}, \mathrm{pH} 7.4\right)$ using $3.5 \mathrm{kDa}$ MWCO dialysis membrane (Spectrumlabs). The protein concentration was determined by the Bradford method. The integrity and purity of chimeric proteins were confirmed by SDS-PAGE. The exact molecular mass of proteins was identified on MALDI-TOF MS as described by Mlynarcik et al. [12].

Affinity purification of anti-OspA and anti-OspC antibodies. The antigen-specific antibodies were affinity purified, as described previously [9]. Briefly, pre-swollen CNBrSepharose $4 \mathrm{~B}$ beads (Pharmacia Fine Chemicals) were mixed with purified his-tagged OspA or OspC proteins of SKT-2, SKT-4 and SKT-7.1 (previously produced in the author's laboratory $[9,13]$ ). Total IgGs (previously purified in the author's laboratory [9]) were incubated with beads for 3 hrs at room temperature, and antigen-specific antibodies eluted from the beads with $3.6 \mathrm{M} \mathrm{MgCl}_{2}$. A small aliquot of eluate was subjected to SDS-PAGE to confirm the purity of isolated antibodies.

Immunospot with antigen-specific antibodies. Chimeric proteins ( $1 \mu \mathrm{g}$ total protein) were spotted on the Immobilone FL membrane. The membrane was blocked with Odyssey Blocking Solution (LI-COR) at $37^{\circ} \mathrm{C}$ for $1 \mathrm{~h}$, and incubated with purified antigen-specific antibodies (anti-OspA or anti-OspC antibody). After five washings with PBS-T (PBS containing $0.1 \%$ Tween 20 ), the membrane was incubated in IRDye 680RD detection reagent (LI-COR; 1:4,000 diluted in Odyssey Blocking Solution) at $37^{\circ} \mathrm{C}$ for $1 \mathrm{~h}$. After washing with PBS-T and PBS, signals were captured at $700 \mathrm{~nm}$ using an Odyssey CLx near-infrared fluorescence imaging system (LI-COR). As negative control, a pool of sera from healthy donors was used. For input control, chimeric antigens were incubated with monoclonal Anti-6X His IgG CF 770 antibody (Sigma Aldrich) diluted 1:5,000 in Odyssey Blocking Solution for $1 \mathrm{hr}$. Recombinant OspA and OspC observed on the membrane was used as a positive control.

Collection of human serum samples and screening with commercial two-tier tests. The total of 169 serum samples from patients with Lyme-like symptoms (skin rash, meningitis, arthritis) were collected from the P. J. Safarik University Hospital in Kosice. The collected sera were pre-screened for the presence/absence of Borrelia-specific antibodies by BORRELIA IgM ELISA and BORRELIA IgG ELISA (Biomedica Immunoassays) before screening with chimera-based ELISA

Lyme disease BORRELIA IgM ELISA and BORRELIA IgG ELISA (Biomedica Immunoassays) were used for the detection of anti-Borrelia antibodies. The tests are polyvalent and were performed according to the manufacturer's instructions. In the case of IgM ELISA, OspC of B. afzelii PKo and B. garinii 20047 (p21), inner part of flagelin of B. bavariensis PBi $(\mathrm{p} 41 / \mathrm{I})$ and fusion proteins of VIsE from different Borrelia 
genospecies were used as antigens. In the case of IgG ELISA, OspC of B. burgdorferi sensu stricto B31 and B. garinii 20047, p18 and p100 of B. afzelii PKo and fusion proteins of VIsE from different Borrelia genospecies were used as antigens. Values between two and three standard deviations were considered equivocal and counted as positive. Immunoblot Borrelia ViraStripe ${ }^{\circledast}$ (Viramed Biotech, Germany) was used for the detection of anti-Borrelia IgMs and IgGs in ELISApositive sera. The test strips carried purified antigens (p83, p58, p43, p30, p21, p14, DbpA, Osp17 and OspC for igG and p41, p39, OspC and Osp17 for IgM detection) from B. afzelii (Pko), B. burgdorferi sensu stricto and recombinant VlsE. Immunoblots were performed and interpreted according to the manufacturer's instructions.

ELISA with chimeric proteins. Solutions of purified chimeric proteins in $140 \mathrm{mM}$ sodium carbonate, $\mathrm{pH} 9.0$, were used to coat the wells of flat-bottomed plates (Nunc). Aliquote $50 \mu \mathrm{l}$ of a solution with concentration $1.0 \mu \mathrm{g} / \mathrm{ml}$ was added to each well, and the microwell plate incubated at $4{ }^{\circ} \mathrm{C}$ overnight. The antigen solution was removed from the wells, the plate was washed three times with $140 \mathrm{mM}$ sodium carbonate ( $\mathrm{pH} 9.0)$ and $200 \mu \mathrm{l}$ of blocking solution ( $2 \%$ bovine serum albumin fraction $\mathrm{V}$ in $140 \mathrm{mM}$ sodium carbonate, $\mathrm{pH} 9.0$ ) was added. After $1 \mathrm{hr}$ of incubation at $37^{\circ} \mathrm{C}$, the plates were washed three times with PBS-T (0.05\% Tween 20, pH 7.5). Serum samples were diluted 1:100 in 2\% fetal bovine serum (Gibco) in PBS, and $50 \mu \mathrm{l}$ of each sample was added to the ELISA plate microwells. After $1 \mathrm{hr}$ of incubation at $37^{\circ} \mathrm{C}$, the wells were washed with PBS-T and Protein A/G conjugated with HRP (Pierce; diluted 1:10,000 in PBS, pH 7.4) was added. Following incubation for $1 \mathrm{~h}$ and washing, $100 \mu \mathrm{l}$ of HRP680 assay buffer (LI-COR) was added to each well. After incubation, stop solution (LI-COR) was added to each well. The absorbance was read at $700 \mathrm{~nm}$ on an Odyssey CLx nearinfrared fluorescence imaging system (LI-COR). As negative controls, five serum samples from healthy individuals from non-endemic area were used. The same negative controls were included in each plate.

Data analysis. The cutoff values of ELISA were calculated as the mean absorbance of the true negative sera plus three times standard deviations. Sensitivity and specificity were calculated according the following formulas:

$\%$ Sensitivity $=[$ True positive $/($ True positive + False negative $)] \times 100$;

$\%$ Specificity $=[$ True negatives $/($ False positives + True negatives) $] \times 100$.

The $\%$ positive predictive value (PPV) is defined as $\mathrm{PPV}=[$ True positive $/($ True positive + False positive $)] \times 100$; The $\%$ negative predictive value (NPV) is defined as $\mathrm{NPV}=[$ True negative $/($ True negative + False negative $)] \times 100$.

For measurement of inter-assay agreement between conventional two-tier test and chimera based ELISA, Cohen's kappa was used.

\section{RESULTS}

Chimeric proteins. Borrelial outer surface proteins OspA and $\mathrm{OspC}$ were the focus of the presented strudy Borrelial outer surface proteins OspA and OspC were the focus of the presented study due to their diagnostic potential. On the basis of the selected epitopes, we synthesized the chimeric genes $(a / c-2, a / c-4$ and $a / c-7.1)$ selected epitopes (OspC E1, OspC $\mathrm{E} 2$, OspC E3 and OspC E5 from OspC and LA-2 epitope from OspA) we designed, synthesized the chimeric genes $(a / c-2, a / c-4$ and $a / c-7.1)$. Genes were ligated into expression vector and electrotransferred into $E$. coli. The transformants were selected in the presence of carbenicillin. The chimeric genes from selected $E$. coli clones were amplified with vector specific primers and analyzed by sequencing.

The protein yield of the recombinant chimeras was approximately $60 \mathrm{mg}$ for both A/C-2 and A/C-4 and $200 \mathrm{mg}$

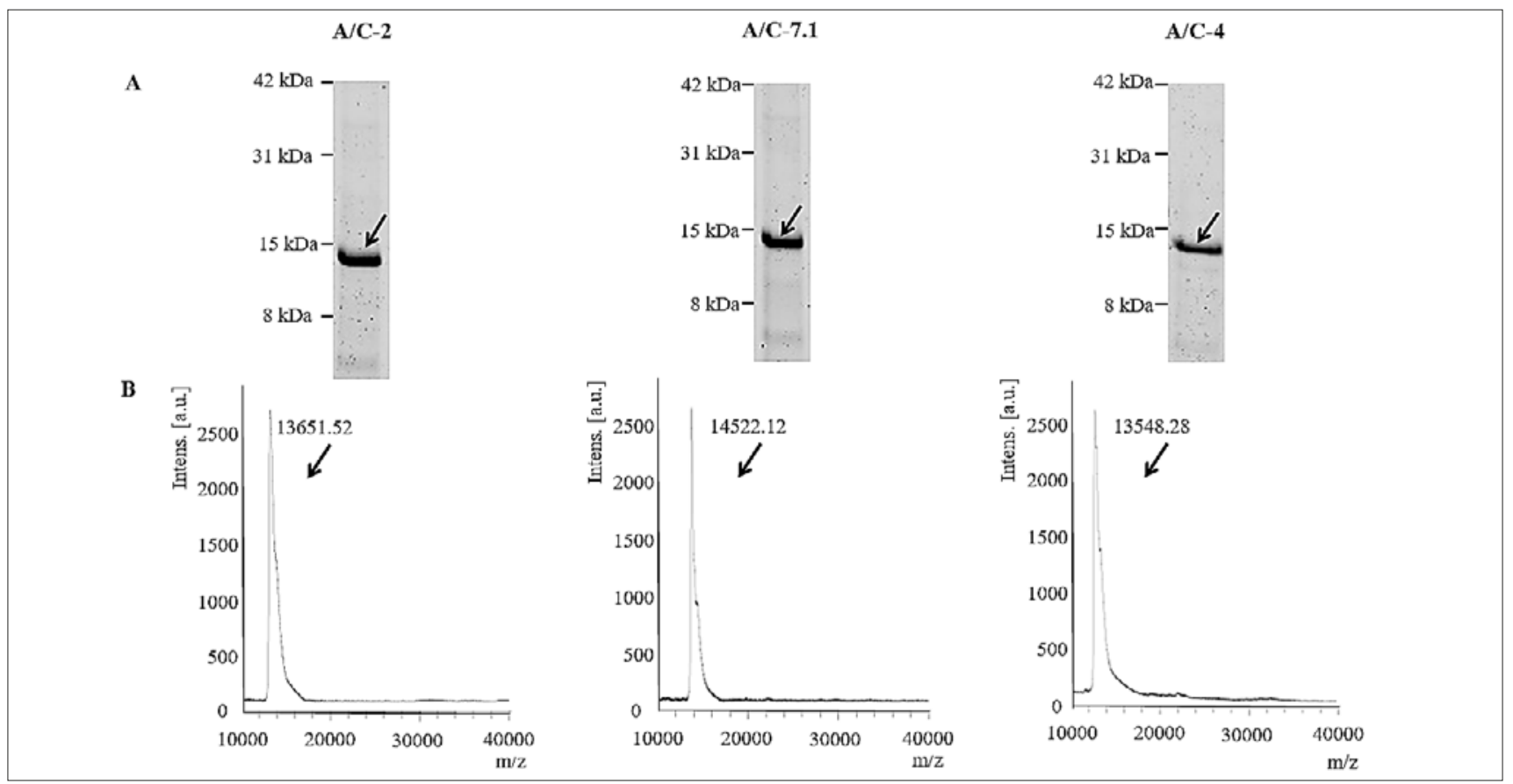

Figure 2. Production of recombinant chimeric proteins. Presence of the recombinant A/C-2 13.6 kDa, A/C-7.1 14.4 kDa and A/C-4 13.5 kDa and molecular weight was confirmed by SDS-PAGE (Panel A) and mass spectrometry (Panel B) 
Table 1. Epitopes selected for design of chimeric proteins

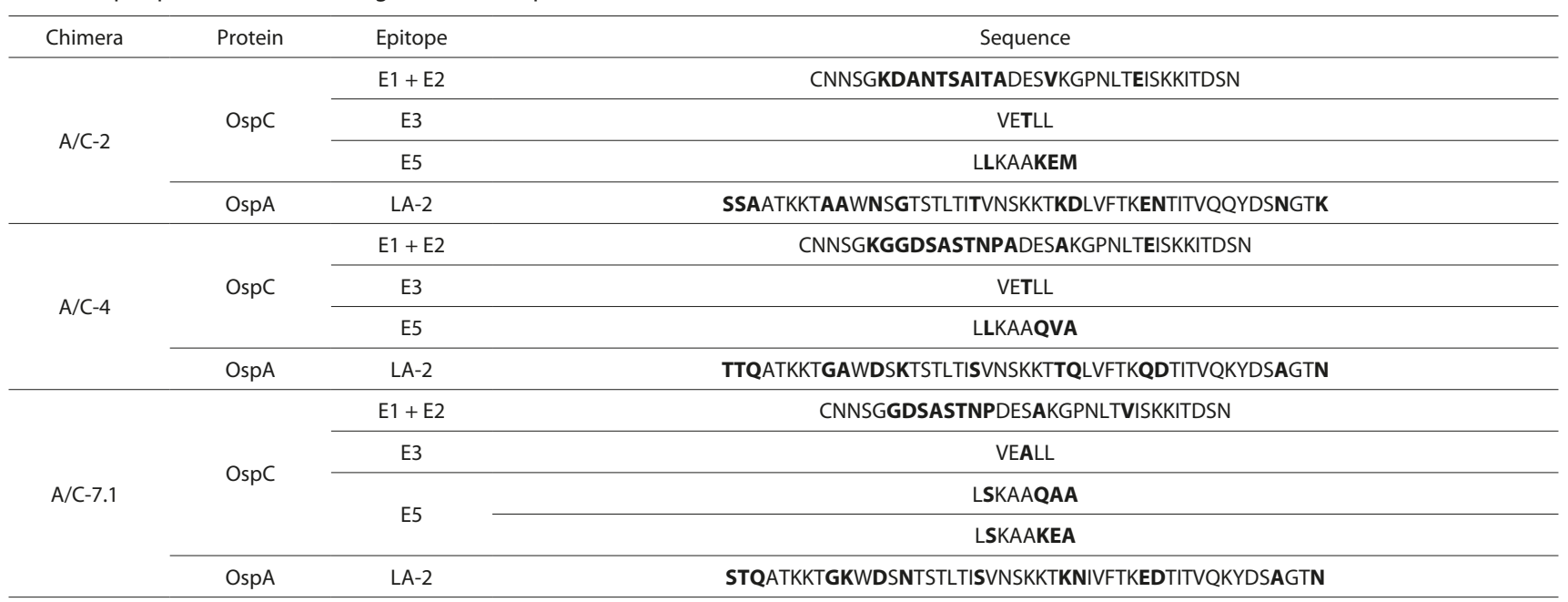

Variable amino acids shown in bold

for A/C-7.1 per liter of medium. Purity of chimeric proteins was verified by SDS-PAGE (Fig. 2, Panel A). The exact molecular weights of his-tagged chimeras were confirmed on the MALDI-TOF, which were in accordance with the masses predicted in silico $(13.6 \mathrm{kDa}$ for $\mathrm{A} / \mathrm{C}-2,14.4 \mathrm{kDa}$ for A/C-7.1 and 13.5 kDa for A/C-4) (Fig. 2, Panel B).

Immunoreactivity of chimeric antigens with antigenspecific antibodies. To demonstrate that epitopes are accessible to paratopes of specific antibodies, the chimeric proteins were tested for reactivity with anti-OspA and antiOspC antibodies. First, the antigen-specific antibodies were purified from total IgGs against recombinant OspC or OspA by affinity chromatography. The A/C-2 and A/C-7.1 chimera showed reactivity on immunoblot with both antigen-specific antibodies, and were subjected to further serological testing (Fig. 3, lines 1 and 3). The chimeric proteins did not show cross-reactivity when incubated with healthy donor sera (Fig. 3, line 2). The chimeric protein A/C-4 lacked immunoreactivity with anti-OspA antibody and was not subjected to further studies.

Screening of human serum samples by commercial assays. Based on the results of commercial assays, 116 serum samples

Table 2. Serum samples used in study and results of immunoassays

\begin{tabular}{lccc}
\hline \multirow{2}{*}{ Clinical symptoms } & \multicolumn{2}{c}{ Positive ELISA results/number of sera tested } \\
\cline { 2 - 4 } & Two-tier test & A/C-2 & A/C-7.1 \\
\hline Skin rash & $22 / 34$ & $25 / 34$ & $23 / 34$ \\
\hline Meningitis & $60 / 93$ & $66 / 93$ & $66 / 93$ \\
\hline Arthritis & $34 / 42$ & $27 / 42$ & $31 / 42$ \\
\hline Total & $\mathbf{1 1 6 / 1 6 9}$ & $\mathbf{1 1 8 / 1 6 9}$ & $\mathbf{1 2 0 / 1 6 9}$ \\
\hline
\end{tabular}

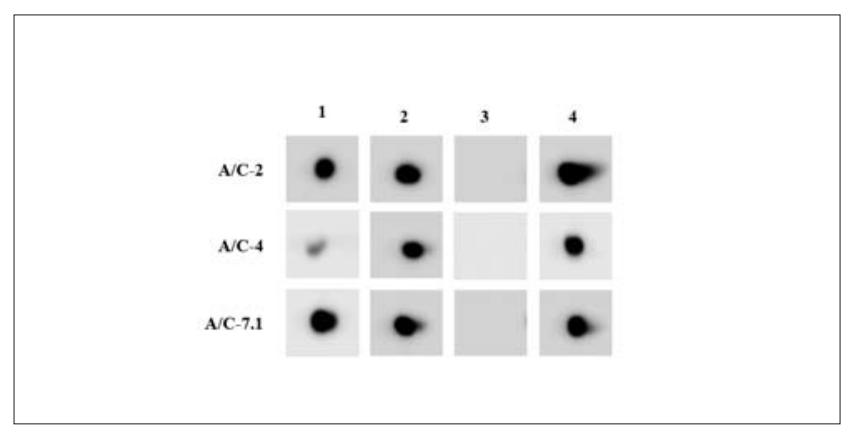

Figure 3. Immuno-reactivity of chimeric protein with anti-OspA and anti-OspC antibody. Overexpressed His-tagged chimeric proteins were separated on SDSPAGE, transferred on membrane and incubated with anti-OspA antibody (line 1) and anti-OspC antibody (line 2) and IRDye 680RD detection reagent. Chimeric protein incubated with healthy donor sera served as negative control (line 3). Membranes incubated with monoclonal Anti-6X His IgG, $\mathrm{CF}^{\mathrm{TM}} 770$ antibody served as an input control (line 4).

were classified into Panel 1 (LD serum samples; 37 sera positive for IgM and 79 sera positive for IgG) and 53 serum samples were classified into Panel 2 (non-LD serum samples; negative for IgM and IgG). Panel 2 represents potentially cross-reactive sera from patients with Lyme-like symptoms (skin rash, meningitis, arthritis) (Tab. 2).

Evaluation of chimera-based ELISAs using pre-screened human sera. Next, the purified chimeric proteins A/C-2 and $\mathrm{A} / \mathrm{C}-7.1$ were evaluated as a diagnostic reagent to detect anti-Borrelia antibodies using this well-characterized serum panels. The chimeric proteins A/C-2 and A/C-7.1 showed sensitivity of $80.17 \%$ and $91.37 \%$, respectively, when tested with serologically characterized serum panels. The 23 serum samples from Panel 1 (116 serologically positive sera) were diagnosed as negative by the A/C-2 ELISA and ten serum

Table 3. Comparison of commercial two-tier test with chimera-based ELISA

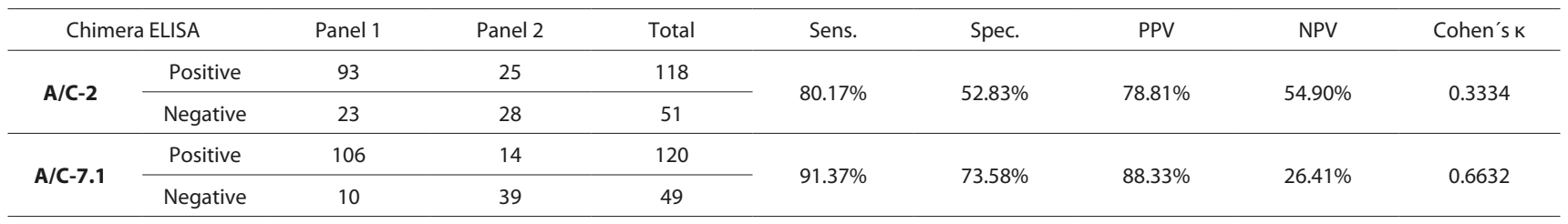


samples were negative by the A/C-7.1 ELISA. Conversely, when testing the chimera-based ELISA on sera from serologically negative patients with Lyme-like symptoms (Panel 2), both chimeras showed immune-reactivity. The 25 serum samples from Panel 2 showed immuno-reactivity in A/C-2 ELISA, and 14 serum samples were negative when A/C-7.1 was used as a capture antigen.

\section{DISCUSSION}

Because of a lack of pathognomonic symptoms, many cases of LD go unreported in areas of endemicity [4]. Accurate and reliable diagnostic assays are essential for planning the treatment as well as to exclude the large majority of uninfected patients with 'Lyme-like' symptoms. The development of universal assays for immunoserologic evaluation of LD is complicated by the presence of three B. burgdorferi sensu lato genospecies in Europe, and significant antigenic variation within each genospecies. Employment of B-cell epitopes from the strains specific for defined geographic areas has the potential to improve the performance of serological diagnosis [4]. Several studies have shown that synthetic peptides (B-cell epitopes) can be used for the serological diagnosis of viral and bacterial as well as parasitic diseases $[14,15]$. To test this hypothesis, in the current study, chimeric proteins were generated containing B-cell epitopes of OspA and OspC of SKT-2 (B. burgdorferi), SKT-7.1 (B. bavariensis) and SKT-4 (B. afzelii) strains endemic in Eastern Slovakia, and their performance was tested in chimera-based assays (chimeras were used as capture antigens) with human serum samples.

The outer surface proteins OspA and OspC selected to construct multi-epitope chimeric proteins are highly immunogenic. The expression of OspA was shown to be downregulated during transmission to mammalian host and thus it is thought that the immune system is not exposed to significant OspA expression after infection [16]. However, weak but detectable antibody response induced by OspA was detected early after infection in mammal hosts $[17,18]$. Disruption of borrelial plasma membrane triggered by the innate immune system can explain the detection of OspA in the serum from patients diagnosed with early LD, but remain at low levels or bound in immune complexes [5]. Schutzer et al. showed that IgM is the predominant isotype of OspA specific antibodies [18]. To our knowledge, this is the first time that OspA was used as antigen in diagnostic immunoassay.

The presented results show that chimeric proteins A/C-2 and A/C-7.1 vary in their immuno-reactivity when tested with sera from patients suspected of $\mathrm{LD}$. Recombinant protein A/C-2 and A/C-7.1 detected anti-Borrelia antibodies in 118 respectively 120 sera from total number of collected sera. Recombinant chimeric protein A/C-2 was more sensitive in patients with skin rash, and produced less positivity when tested with arthritis sera, which may reduce repeated drug use for false-positive patients. Compared with the results obtained from commercial immunoassays, the application of recombinant multi-epitope chimeric proteins from strains specific for Slovakia as diagnosis antigens, produced fair agreement in the case of A/C-2 $(0.20<\kappa<0.40)$, and good agreement $(0.60<\kappa<0.80)$ when $\mathrm{A} / \mathrm{C}-7.1$ was used as the capture antigen. The 70 sera were tested positive in all assays.
The infectious and immunopathological conditions (viral infections, bacterial endocarditis, rheumatoid arthritis, and systemic lupus erythematosus) can compromise the accuracy of diagnostic assays [19]. Significant differences in the sensitivity and specificity of assays used to detect anti-Borrelia antibodies can be found when the results from different laboratories are compared $[20,21]$. It was shown that results can be influenced by the choice of diagnostic assays (different antigens used for serological testing) and ELISA-immunoblot combination in the two-tier testing system [21]. Employment of single antibody binding regions (epitopes) of immunogenic proteins could remove crossreactive epitopes, and has the potential to achieve higher specificity of diagnostic immunoassays.

The combination of multiple specific B-cell epitopes from antigenic borrelial proteins may increase the sensitivity and specificity of serologic assays. The chimeric proteins showed good sensitivity in diagnosis of LD infection and have the potential to improve the diagnosis of LD. In this study, it was also noted that the inclusion of the LA-2 epitope of OspA protein in the recombinant multi-epitope antigens may have enhanced the diagnostic accuracy of the serologic assays; however, further studies are required to address this hypothesis.

\section{Acknowledgements}

The study was supported by VEGA 1/0261/15, VEGA $1 / 0258 / 15$ and APVV-14-0218. MALDI-TOF used in this study was funded by the EU infrastructure project INFEKTZOON - 26220120002.

\section{REFERENCES}

1. Wilske B, Fingerle V, Schulte-Spechtel U. Microbiological and serological diagnosis of Lyme borreliosis. FEMS Immunol Med Microbiol. 2007; 49(1): 13-21.

2. Steere AC, McHugh G, Damle N, Sikand VK. Prospective study of serologic tests for lyme disease. Clin Infect Dis. 2008; 47(2): 188-95.

3. Aguero-Rosenfeld ME, Wormser GP. Lyme disease: diagnostic issues and controversies. Expert Rev Mol Diagn. 2015; 15(1): 1-4.

4. Reed KD. Laboratory testing for Lyme disease: possibilities and practicalities. J Clin Microbiol. 2002; 40(2): 319-24.

5. Cheung CS, Anderson KW, Benitez KY, Soloski MJ, Aucott JN, Phinney KW et al. Quantification of Borrelia burgdorferi Membrane Proteins in Human Serum: A New Concept for Detection of Bacterial Infection. Anal Chem. 2015; 87(22): 11383-8.

6. Hsieh YF, Liu HW, Hsu TC, Wei JC, Shih CM, Krause PJ et al. Serum reactivity against Borrelia burgdorferi OspA in patients with rheumatoid arthritis. Clin Vaccine Immunol. 2007; 14(11): 1437-41.

7. Ding W, Huang X, Yang X, Dunn JJ, Luft BJ, Koide S et al. Structural identification of a key protective B-cell epitope in Lyme disease antigen OspA. J Mol Biol. 2000; 302(5): 1153-64.

8. Aguero-Rosenfeld ME, Nowakowski J, Bittker S, Cooper D, Nadelman $\mathrm{RB}$, Wormser GP. Evolution of the serologic response to Borrelia burgdorferi in treated patients with culture-confirmed erythema migrans. J Clin Microbiol. 1996; 34(1): 1-9.

9. Pulzova L, Flachbartova Z, Bencurova E, Potocnakova L, Comor L, Schreterova E et al. Identification of B-cell epitopes of Borrelia burgdorferi outer surface protein $\mathrm{C}$ by screening a phage-displayed gene fragment library. Microbiol Immunol. 2016; 60(10): 669-677.

10. Tilly K, Rosa PA, Stewart PE. Biology of infection with Borrelia burgdorferi. Infect Dis Clin North Am. 2008; 22(2): 217-34, v.

11. Robertson J, Guy E, Andrews N, Wilske B, Anda P, Granstrom M et al. A European multicenter study of immunoblotting in serodiagnosis of lyme borreliosis. J Clin Microbiol. 2000; 38(6): 2097-102.

12. Mlynarcik P, Pulzova L, Bencurova E, Kovac A, Dominguez MA, Hresko $S$ et al. Deciphering the interface between a CD40 receptor and borrelial ligand OspA. Microbiol Res. 2015; 170: 51-60. 
13. Pulzova L, Kovac A, Mucha R, Mlynarcik P, Bencurova E, Madar M et al. OspA-CD40 dyad: ligand-receptor interaction in the translocation of neuroinvasive Borrelia across the blood-brain barrier. Sci Rep. 2011; $1: 86$.

14. Alcaro MC, Peroni E, Rovero P, Papini AM. Synthetic peptides in the diagnosis of HIV infection. Curr Protein Pept Sci. 2003; 4(4): 285-90.

15. Maksimov P, Zerweck J, Maksimov A, Hotop A, Gross U, Pleyer U et al. Peptide microarray analysis of in silico-predicted epitopes for serological diagnosis of Toxoplasma gondii infection in humans. Clinical and Vaccine Immunology. 2012; 19(6): 865-74.

16. Yang XF, Pal U, Alani SM, Fikrig E, Norgard MV. Essential role for OspA/B in the life cycle of the Lyme disease spirochete. J Exp Med. 2004; 199(5): 641-8.

17. Wagner B, Freer H, Rollins A, Garcia-Tapia D, Erb HN, Earnhart C et al. Antibodies to Borrelia burgdorferi OspA, OspC, OspF, and C6 antigens as markers for early and late infection in dogs. Clin Vaccine Immunol. 2012; 19(4): 527-35.
18. Schutzer SE, Coyle PK, Dunn JJ, Luft BJ, Brunner M. Early and specific antibody response to OspA in Lyme Disease. J Clin Invest. 1994; 94(1): 454-7.

19. Gomes-Solecki MJ, Dunn JJ, Luft BJ, Castillo J, Dykhuizen DE, Yang $\mathrm{X}$ et al. Recombinant chimeric Borrelia proteins for diagnosis of Lyme disease. J Clin Microbiol. 2000; 38(7): 2530-5.

20. Bakken LL, Callister SM, Wand PJ, Schell RF. Interlaboratory comparison of test results for detection of Lyme disease by 516 participants in the Wisconsin State Laboratory of Hygiene/College of American Pathologists Proficiency Testing Program. J Clin Microbiol. 1997; 35(3): 537-43.

21. Ang CW, Notermans DW, Hommes M, Simoons-Smit AM, Herremans T. Large differences between test strategies for the detection of antiBorrelia antibodies are revealed by comparing eight ELISAs and five immunoblots. Eur J Clin Microbiol Infect Dis. 2011; 30(8): 1027-32. 